\title{
Planktonic diatoms in lotic and lentic environments in the Lago dos Tigres hydrologic system (Britânia, Goiás, Brazil): Coscinodiscophyceae and Fragilariophyceae
}

\author{
BÁRBARA DUNCK ${ }^{1,4}$, INA DE SOUZA NOGUEIRA² and \\ MARIA DAS GRAÇAS MACHADO ${ }^{3}$
}

(received: October 21, 2011; accepted: March 23, 2012)

\begin{abstract}
Planktonic diatoms in lotic and lentic environments in the Lago dos Tigres hydrologic system (Britânia, Goiás, Brazil): Coscinodiscophyceae and Fragilariophyceae). A floristic study of Coscinodiscophyceae and Fragilariophyceae in the Lago dos Tigres hydrologic system is presented. Phytoplankton samples were collected with a plankton net $(25 \mu \mathrm{m}$ mesh) at 11 sampling stations, three in lotic areas and eight in lacustrine zones. Eleven species of Coscinodiscophyceae and eight of Fragilariophyceae were identified; descriptions, comments, their geographic distributions for the midwestern region of Brazil, type of environment (lentic/lotic), and illustrations are provided. Among the taxa found, 13 were recorded for the first time in midwestern Brazil: Aulacoseira alpigena, A. distans, A. granulata var. angustissima, A. granulata var. valida, A. muzzanensis, Discostella stelligera var. stelligera, Fragilaria capucina var. fragilarioides, F. delicatissima var. delicatissima, F. javanica, Pleurosira laevis, Staurosira acutirostrata, Staurosirella crassa, and Ulnaria ulna.
\end{abstract}

Key words - algae, Bacillariophyta, inventory, phytoplankton

\section{INTRODUCTION}

The Lago dos Tigres hydrologic system is located near the city of Britânia, in Goiás State, Brazil, and is composed of both lotic and lentic environments (Nabout \& Nogueira 2007). The lake and the surrounding areas are used for tourism and cattle ranching and are of significant regional importance (Geogoiás 2002). The regional landscape is composed in large part of pasturelands with many environmentally degraded areas (JE Oliveira, unpublished data).

Diatoms (Bacillariophyta) are found in all types of aquatic environments (Round 1991, Potapova \& Charles 2003), and according to Round (1991), approximately 260 genera and approximately 100,000 species of diatoms are currently known worldwide. According to Ferrari \& Ludwig (2007), little is known about the diatomaceous flora of Brazil, especially in light of the enormous extensions of its freshwater and ocean areas, and most studies have been concentrated

1. Programa de Pós-Graduação em Ecologia de Ambientes Aquáticos Continentais, Universidade Estadual de Maringá, Maringá, PR, Brazil.

2. Universidade Federal de Goiás, Instituto de Ciências Biológicas, Departamento de Biologia Geral, sala 201a, Campus II, Caixa Postal 131, 74001-970 Goiânia, GO, Brazil.

3. Universidade de Brasília, Instituto de Ciências Biológicas, Departamento de Botânica, ICC Sul, Caixa Postal 4457, 70919-970 Brasília, DF, Brazil.

4. Corresponding author: dunck.barbara@gmail.com in the southern and southeastern regions of that country.

Important studies addressing the taxonomy of the classes Coscinodiscophyceae and Fragilariophyceae have been undertaken in Brazil in the last 10 years in different aquatic ecosystems. A study by Tremarin et al. (2011) of periodically flooded areas evaluated the ultrastructure and distribution of Aulacoseira gessneri (Hustedt) Simonsen in the Pantanal region of Mato Grosso State, and Fontana \& Bicudo (2009) analyzed the classes Coscinodiscophyceae and Fragilariophyceae in superficial sediments in a chain of reservoirs along the Paranapanema River (in the states of São Paulo SP and Paraná - PR). A number of studies of lotic ecosystems have been undertaken, including: Ferrari \& Ludwig (2007), who studied the periphytes and planktonic taxa in the Ivaí River basin (PR); Landucci \& Ludwig (2005), who evaluate these same two classes among planktonic and periphytic species in the rivers of coastal hydrographic basins in Paraná State and reported Aulacoseira distans var. distans (Ehrenberg) Simonsen for the first time in that region; and Brassac \& Ludwig (2003), who surveyed the planktonic taxa of the family Fragilariaceae in the Iguaçu River basin (PR) and identified two new taxa for that state. Likewise, a number of studies have been undertaken in lentic ecosystems, including: Ludwig et al. (2004), who analyzed periphytic species of the class Coscinodiscophyceae found in lakes in the Taim hydrological system (RS) and reported four new occurrences for Rio Grande do Sul State (RS); 
and Morandi et al. (2006), who analyzed the taxa of Coscinodiscophyceae present in the plankton and among periphytes in lakes and streams in the Fontes do Ipiranga State Park Biological Reserve in the city of São Paulo (SP).

Representatives of the class Coscinodiscophyceae do not have a raphe as their principal characteristic, and are found in freshwater as well as saltwater areas. The taxonomy of this class has undergone recent modifications, with the proposal of new genera such as Discostella (Houk \& Klee 2004). The most frequently cited genus in Brazil is Aulacoseira, which has roughly 25 species (most of which have been found in that country) (Ludwig \& Bigunas 2006).

Fragilariophyceae diatoms are important components of both planktonic and periphytic communities in freshwater lakes (Morales 2001). The organisms belonging to the Fragilaria complex within the class Fragilariophyceae demonstrate significant morphological and ecological diversity, and have the capacity to either fix themselves to the substrate or float in the water column (Morales 2001). The taxonomy of this group has undergone substantial changes in the last two decades due to the wider use of electron microscopy to observe the ultrastructural characteristics of the valves (Morales 2001). This technological advance has resulted in the partitioning of Fragilaria into seven different freshwater genera as well as a new circumscription of Fragilaria (Williams \& Round 1987, Round et al. 1990), and in the last 10 years a number of new genera and species have been proposed for this group (Morales 2001, 2005, Morales \& Le 2005, Morales et al. 2010).

Given only the small numbers of studies of diatoms undertaken in Brazil, and the lack of taxonomic investigation of Coscinodiscophyceae and Fragilariophyceae in Goiás State and in the midwestern region of Brazil, the present work sought to inventory the taxa of these classes among the planktonic flora of the Lago dos Tigres hydrologic system during the lowwater period in 2004.

\section{MATERIAL AND METHODS}

Descriptions of the study area and the sampling points in the Lago dos Tigres hydrologic system (Britânia, GO, Brazil, $15^{\circ} 36^{\prime} 33.5^{\prime \prime}-15^{\circ} 22^{\prime} 8.7^{\prime \prime} \mathrm{S}$ and $51^{\circ} 17^{\prime} 56.3^{\prime \prime}$ $51^{\circ} 16^{\prime} 91$ ” W) can be found in Nabout \& Nogueira (2007). Although the area is popularly known as a lake, it can be more accurately described as a river valley with choked drainage comprising both lotic (the Água Limpa River that drains into the Vermelho River) and lentic environments (Lago dos Tigres).

Eleven collection stations were established (three in lotic areas and eight in lacustrine zones) and their limnological variables $(\mathrm{pH}$, transparency, water temperature, electrical conductivity, dissolved oxygen, total solids, total nitrogen, and total phosphorus) and morphometric parameters (width of the body of water and its depth) were evaluated. All of these abiotic variables can be found in Nabout \& Nogueira (2007); these authors described this system as being mesotrophic to oligotrophic, with higher concentrations of nutrients during the dry season.

Phytoplankton samples were collected in the surface waters during the dry season (June to September/2004) and at the beginning of the rainy season (October to November/2004) using plankton nets ( $25 \mu \mathrm{m}$ mesh). The samples were fixed in Transeau solution (Bicudo \& Menezes 2006) for qualitative studies and subsequently deposited in the herbarium at the Universidade Federal de Goiás.

The planktonic material was oxidized using the technique described by Simonsen (1974), as modified by Moreira Filho \& Valente-Moreira (1981); permanent slides were prepared using Naphrax resin. Qualitative analyses were undertaken using a Zeiss Axioscop 40 optical microscope. Scanning electron microscopy (SEM) samples were prepared according to Ferrario et al. (1995). Samples of the oxidized material were deposited on coverslips and dehydrated by warming them; the coverslips were subsequently secured to metallic supports using carbon tape, sputter coated with gold, and observed under a Jeol model JSM 6610 scanning electron microscope. The classification system utilized follows Round et al. (1990), and the identifications of the species were based on the specific literature (Krammer \& Lange-Bertalot 1997, Ludwig \& Flôres 1997, Metzeltin \& Lange-Bertalot 1998, Morales 2001, 2005, Brassac \& Ludwig 2003, Houk \& Klee 2004, Landucci \& Ludwig 2005, Ferrari \& Ludwig 2007, Fontana \& Bicudo 2009, Morales et al. 2010).

\section{RESULTS AND DISCUSSION}

Floristic studies of the classes Coscinodiscophyceae and Fragilariophyceae in the Lago dos Tigres hydrologic system resulted in the identification of 19 infrageneric taxa, of which 11 belonged to Coscinodiscophyceae and eight to Fragilariophyceae. Among the taxa inventoried, 13 were cited for the first time for the midwestern region of Brazil: Aulacoseira alpigena, A. distans, A. granulata var. angustissima, A. granulata var. valida, A. muzzanensis, Discostella stelligera var. stelligera, Fragilaria capucina var. fragilarioides, F. delicatissima var. delicatissima, F. javanica, Pleurosira laevis, Staurosira acutirostrata, Staurosirella crassa, and Ulnaria ulna. 


\section{DIVISION BACILLARIOPHYTA}

Class Coscinodiscophyceae

Order Aulacoseirales Crawford

Family Aulacoseiraceae Crawford

Aulacoseira Thwaites

Aulacoseira alpigena (Grunow) Krammer, Nova Hedwigia 52: 93. 1991.

Figures 1-2

Frustules cylindrical in pleural view, forming short chains, straight, united by conspicuous linking spines. Delicate areola striae slightly oblique in relation to the pervalvar axis. Height: $3.5-4 \mu \mathrm{m}$; diameter: $4-5 \mu \mathrm{m}$; 14-16 striae in $10 \mu \mathrm{m} ; 18-20$ areolae in $10 \mu \mathrm{m}$.

Short linking spines and slightly oblique striae are characteristics of this species (Krammer \& LangeBertalot 1991). The specimens analyzed resembled those illustrated for the species by Krammer \& LangeBertalot (1997, page 292, plate 31, figures 1, 14 and 15) and those of the first circumscription of the species (Melosira distans var. alpigena Grunow in Van Heurck) illustrated by Hubber- Pestalozzi (1942, page 392, plate XVI, figure $477 \mathrm{~g}$ ). This species was found only in lotic environments.

Geographical distribution in the midwestern Brazil: first record.

Occurrence in the samples: BRAZIL. GoIÁs: Britânia, Rio Vermelho, Station 10, plankton, 14-VII2004, Nogueira et al. 906 (UFG29889, permanent slide); Rio Água Limpa, Station 1, plankton, 14-VII-2004, Nogueira et al. 908 (UFG29891, permanent slide).

Aulacoseira ambigua (Grunow) Krammer var. ambigua, Bacillaria 2: 56. 1979.

Figures 3, 33-34

Frustules cylindrical, forming straight chains, united by short spines, distinct. Areola striae obliquely arranged in relation to the pervalvar axis. Height: 5-12 $\mu \mathrm{m}$; diameter: 5-7 $\mu \mathrm{m}$; 15-16 striae in $10 \mu \mathrm{m}$; 14-19 areolae in $10 \mu \mathrm{m}$.

Aulacoseira ambigua was recorded in lentic and lotic environments in the present study.

Geographical distribution in midwestern Brazil: Planaltina (Souza \& Moreira Filho 1999).

Occurrence in the samples: BRAZIL. GoIÁs: Britânia, Rio Vermelho, Station 10, plankton, 14-VII2004, Nogueira et al. 906 (UFG29889, permanent slide); Rio Água Limpa, Station 1, plankton, 14-VII-2004, Nogueira et al. 908 (UFG29891, permanent slide); Rio Vermelho, Station 11, plankton, 05-VI-2004, Nogueira et al. 881 (UFG29870, permanent slide); Lago dos Tigres, Station 6, plankton, 09-IX-2004, Nogueira et al. 989 (UFG29911, permanent slide); Lago dos Tigres, Station 7, plankton, 04-VI-2004, Nogueira et al. 878 (UFG29867, permanent slide); Lago dos Tigres, Station 7, plankton, 13-VII-2004, Nogueira et al. 903 (UFG29886, permanent slide); Lago dos Tigres, Station 6, plankton, 19-VIII-2004, Nogueira et al. 948 (UFG29898, permanent slide); Rio Vermelho, Station 11, plankton, 05-VI-2004, Nogueira et al. 882 (UFG29871, permanent slide); Rio Vermelho, Station 10, plankton, 05-VI-2004, Nogueira et al. 880 (UFG29880, permanent slide); Rio Vermelho, Station 11, plankton, 14-VII-2004, Nogueira et al. 907 (UFG29890, permanent slide); Lago dos Tigres, Station 6, plankton, 09-IX-2004, Nogueira et al. 990 (UFG29912, permanent slide); Lago dos Tigres, Station 6, plankton, 16-X-2004, Nogueira et al. 1028 (UFG29923, permanent slide).

Aulacoseira distans (Ehrenberg) Simonsen, Bacillaria 2: 57.1979.

Figures 4, 35

Frustules cylindrical, forming straight chains, short, united by short spines. Areolate striae parallel in relation to the pervalvar axis, although some valves with slightly oblique striae. Height: 7-8 $\mu \mathrm{m}$; diameter: 19-21 $\mu \mathrm{m}$; $10-11$ striae in $10 \mu \mathrm{m}$; $17-18$ areolae in $10 \mu \mathrm{m}$.

The specimens analyzed had characteristics similar to those described and illustrated for the first circumscription of the species (Melosira distans (Ehrenberg) Kützing) by Hubber-Pestalozzi (1942, page 392, plate XVI, part 2, figure 477i) and Hustedt (1976, page 92, figure 53). Aulacoseira distans has morphological characteristics similar to A. muzzaensis (Meister) Krammer (Tremarin et al. 2011); although $A$. distans has smaller measurements for the pervalvar axis than A. muzzaensis (wider than long) it does have slightly oblique striae on some valves (Hustedt 1976, Krammer \& Lange-Bertalot, 1991). This species was encountered in lentic and lotic environments.

Geographical distribution in midwestern Brazil: first record.

Occurrence in the samples: BRAZIL. GoIÁs: Britânia, Lago dos Tigres, Station 8, plankton, 13-VII2004, Nogueira et al. 904 (UFG29887, permanent slide); Lago dos Tigres, Station 7, plankton, 13-VII-2004, Nogueira et al. 903 (UFG29886, permanent slide); Lago dos Tigres, Station 7, plankton, 04-VI-2004, Nogueira et al. 878 (UFG29867, permanent slide); Lago dos Tigres, Station 4, plankton, 04-VI-2004, Nogueira et al. 

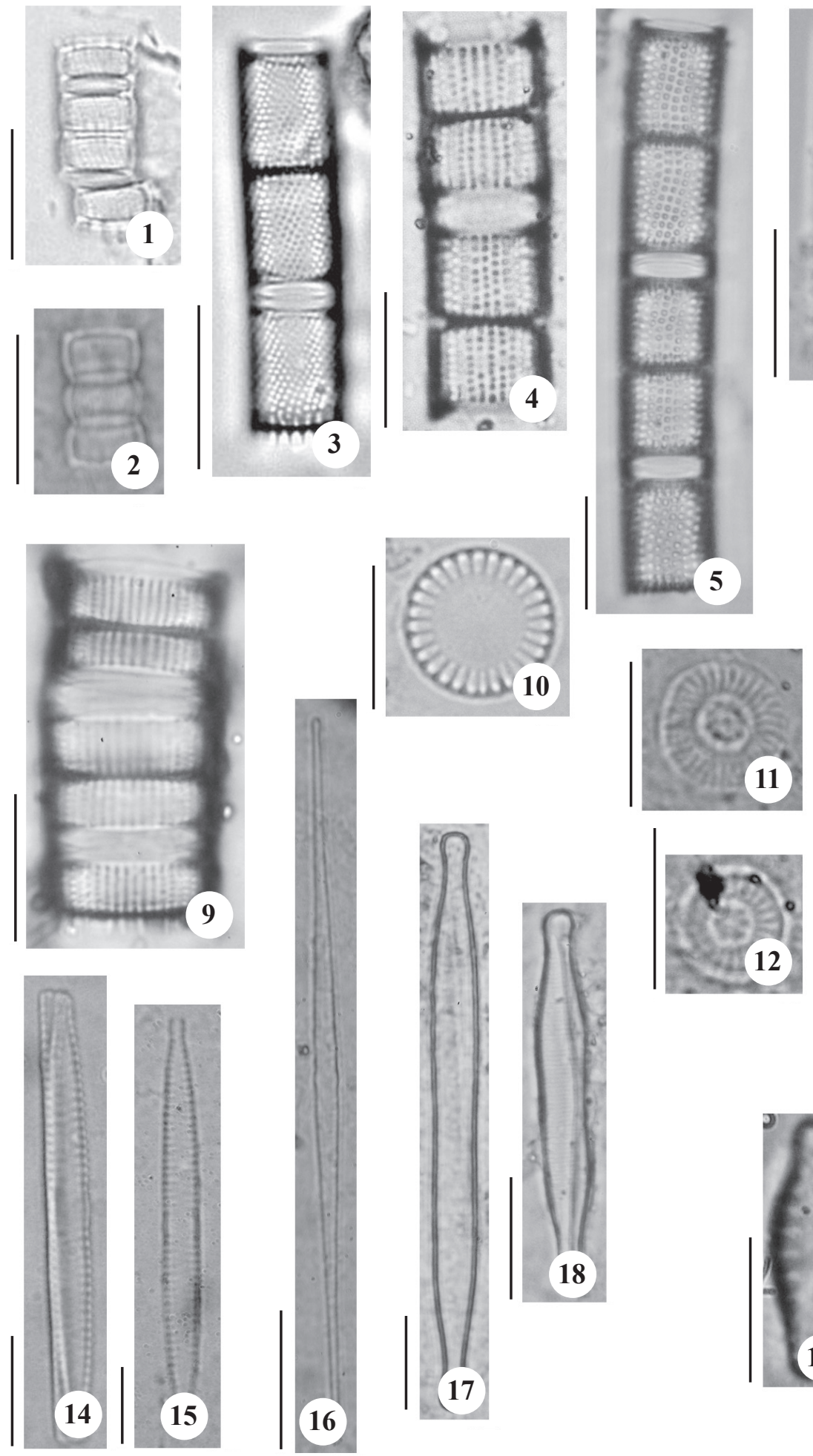

16

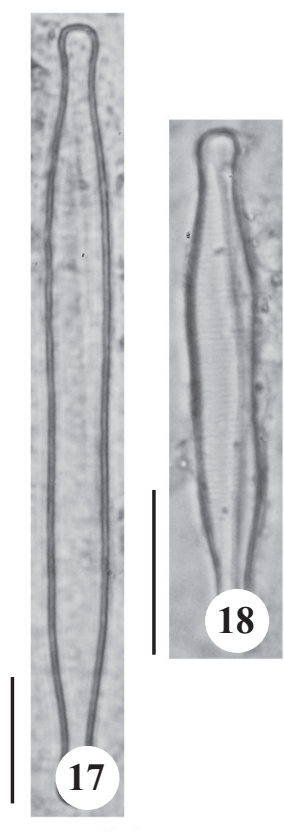

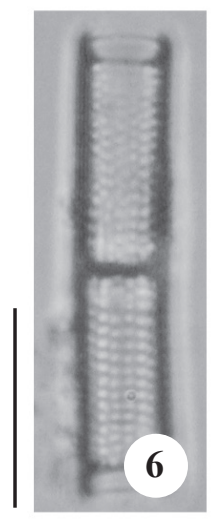
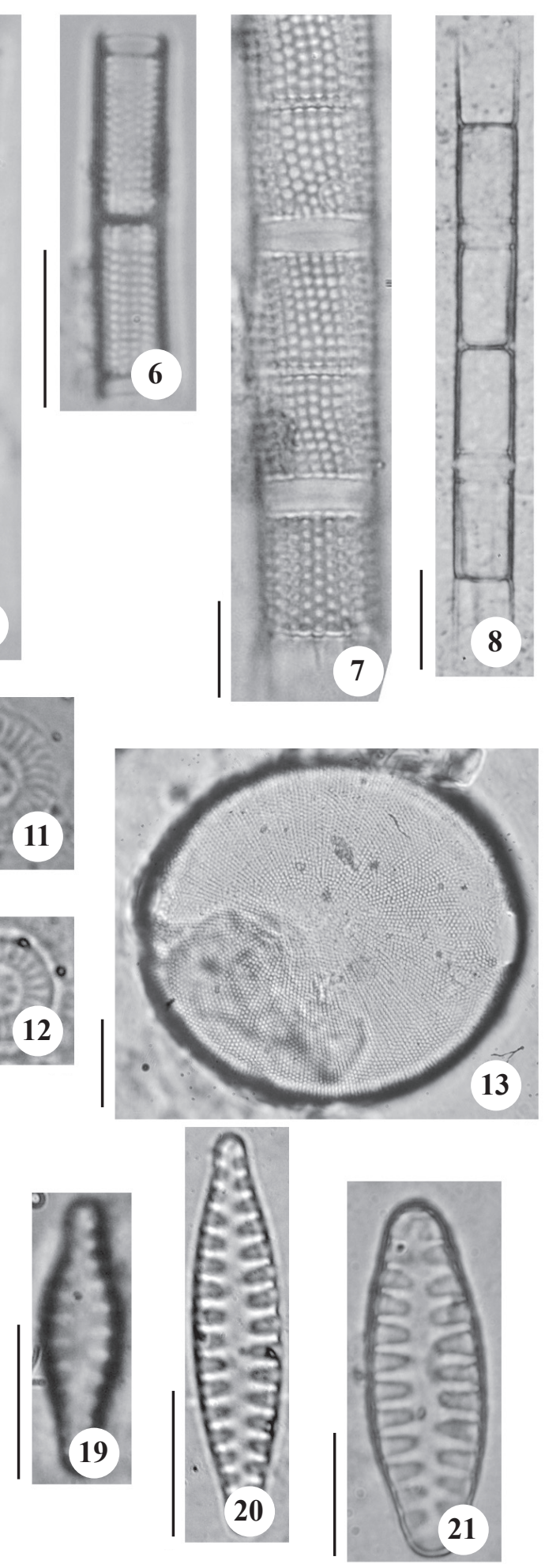

Figures 1-21. Planktonic Coscinodiscophyceae and Fragilariophyceae in the Lago dos Tigres hydrologic system, Goiás, Brazil. Valvar view using an optical microscope. 1-2. Aulacoseira alpigena. 3. Aulacoseira ambigua var. ambigua. 4. Aulacoseira distans. 5. Aulacoseira granulata var. granulata. 6. Aulacoseira granulata var. angustissima. 7. Aulacoseira granulata var. valida. 8. Aulacoseira herzogii. 9. Aulacoseira muzzanensis. 10. Cyclotella meneghiniana. 11-12. Discostella stelligera var. stelligera. 13. Pleurosira laevis. 14-15. Fragilaria capucina var. fragilarioides. 16. Fragilaria delicatissima var. delicatissima. 17-18. Fragilaria javanica. 19-20. Staurosira acutirostrata. 21. Staurosirella crassa. Bar $=10 \mu \mathrm{m}$. 
877 (UFG29866, permanent slide); Lago dos Tigres, Station 6, plankton, 09-IX-2004, Nogueira et al. 989 (UFG29911, permanent slide); Lago dos Tigres, Station 6, plankton, 09-IX-2004, Nogueira et al. 990 (UFG29912, permanent slide); Lago dos Tigres, Station 6, plankton, 16-X-2004, Nogueira et al. 1028 (UFG29923, permanent slide); Rio Água Limpa, Station 1, plankton, 16-X-2004, Nogueira et al. 1023 (UFG29918, permanent slide); Rio Vermelho, Station 11, plankton, 06-XI-2004, Nogueira et al. 1125 (UFG29939, permanent slide); Lago dos Tigres, Station 9, plankton, 06-XI-2004, Nogueira et al. 1123 (UFG29937, permanent slide).

Aulacoseira granulata (Ehrenberg) Simonsen var. granulata, Bacillaria 2: 58. 1979.

Figure 5

Frustules cylindrical in pleural view, forming straight chains united by short linking spines, with both short and long separations. Areola striae roughly parallel and slightly oblique in relation to the pervalvar axis. Height: 13-20 $\mu \mathrm{m}$; diameter: 5-12 $\mu \mathrm{m}$; 14 striae in $10 \mu \mathrm{m}$; 11-12 areolae in $10 \mu \mathrm{m}$.

The presence of long spines is an important characteristic for the taxonomy of the Aulacoseira granulata complex at the species level. The specimens analyzed were similar to those illustrated by Metzeltin et al. (2005, page 244, plate 245, figures 1-4) for Uruguay, this being a species commonly encountered throughout Brazil (Ludwig \& Bigunas 2006). In the present study it was found in both lentic and lotic environments.

Geographical distribution in midwestern Brazil: Rio Araguaia (Nabout et al. 2006).

Occurrence in the samples: BRAZIL. GoIÁs: Britânia, Rio Vermelho, Station 11, plankton, 06-XI2004, Nogueira et al. 1125 (UFG29939, permanent slide); Lago dos Tigres, Station 7, plankton, 04-VI-2004, Nogueira et al. 878 (UFG29867, permanent slide); Rio Vermelho, Station 11, plankton, 05-VI-2004, Nogueira et al. 881 (UFG29870, permanent slide); Lago dos Tigres, Station 6, plankton, 16-X-2004, Nogueira et al. 1028 (UFG29923, permanent slide); Lago dos Tigres, Station 7, plankton, 16-X-2004, Nogueira et al. 1029 (UFG29924, permanent slide); Lago dos Tigres, Station 8, plankton, 16-X-2004, Nogueira et al. 1030 (UFG29925, permanent slide); Lago dos Tigres, Station 8, plankton, 13-VII-2004, Nogueira et al. 904 (UFG29887, permanent slide); Rio Vermelho, Station 10, plankton, 14-VII-2004, Nogueira et al. 906 (UFG29889, permanent slide); Rio Vermelho, Station 11, plankton, 14-VII-2004, Nogueira et al. 907 (UFG29890, permanent slide); Rio Água
Limpa, Station 1, plankton, 14-VII-2004, Nogueira et al. 908 (UFG29891, permanent slide); Lago dos Tigres, Station 9, plankton, 17-X-2004, Nogueira et al. 1031 (UFG29926, permanent slide); Rio Vermelho, Station 10, plankton, 17-X-2004, Nogueira et al. 1032 (UFG29927, permanent slide); Lago dos Tigres, Station 5, plankton, 04-VI-2004, Nogueira et al. 878 (UFG29867, permanent slide); Lago dos Tigres, Station 6, plankton, 09-IX-2004, Nogueira et al. 989 (UFG29911, permanent slide); Lago dos Tigres, Station 2, plankton, 13-VII-2004, Nogueira et al. 898 (UFG29880, permanent slide); Rio Água Limpa, Station 1, plankton, 16-X-2004, Nogueira et al. 1023 (UFG29918, permanent slide); Lago dos Tigres, Station 8, plankton, 19-VIII-2004, Nogueira et al. 950 (UFG29900, permanent slide); Rio Vermelho, Station 11, plankton, 20-VIII-2004, Nogueira et al. 953 (UFG29903, permanent slide); Rio Água Limpa, Station 1, plankton, 20-VIII-2004, Nogueira et al. 954 (UFG29904, permanent slide); Lago dos Tigres, Station 6, plankton, 19-VIII-2004, Nogueira et al. 948 (UFG29898, permanent slide); Rio Vermelho, Station 10, plankton, 06-XI-2004, Nogueira et al. 1124 (UFG29938, permanent slide); Rio Vermelho, Station 10, plankton, 06-XI-2004, Nogueira et al. 1123 (UFG29937, permanent slide); Lago dos Tigres, Station 2, plankton, 05-XI-2004, Nogueira et al. 1116 (UFG29930, permanent slide).

Aulacoseira granulata var. angustissima (O.Müller) Simonsen, Bacillaria 2: 58. 1979.

Figure 6

Frustules cylindrical, forming straight chains united by short linking spines, with short and long separations. Areola striae parallel and slightly oblique in relation to the pervalvar axis. Height: 13-16 $\mu \mathrm{m}$; diameter: 2-4 $\mu \mathrm{m}$; $12-13$ striae in $10 \mu \mathrm{m} ; 12-14$ areolae in $10 \mu \mathrm{m}$.

Low valvar diameter values and delicate striations are characteristic of this species (Hustedt 1976). The specimens were similar to those illustrated by Metzeltin et al. (2005, page 244, plate 245, figure 7) and by Hustedt (1976, page 88, figure 45), and they were encountered in both lentic and lotic environments.

Geographical distribution in midwestern Brazil: first record.

Occurrence in the samples: BRAZIL. GoIÁs: Britânia, Rio Vermelho, Station 11, plankton, 06-XI-2004, Nogueira et al. 1125 (UFG29939, permanent slide); Rio Vermelho, Station 10, plankton, 05-VI-2004, Nogueira et al. 880 (UFG29869, permanent slide); Lago dos Tigres, Station 4, plankton, 04-VI-2004, Nogueira et al. 877 (UFG29866, permanent slide); Rio Vermelho, Station 11, 
plankton, 05-VI-2004, Nogueira et al. 882 (UFG29871, permanent slide); Lago dos Tigres, Station 6, plankton, 16-X-2004, Nogueira et al. 1028 (UFG29923, permanent slide); Lago dos Tigres, Station 5, plankton, 16-X-2004, Nogueira et al. 1027 (UFG29922, permanent slide); Lago dos Tigres, Station 7, plankton, 16-X-2004, Nogueira et al. 1029 (UFG29924, permanent slide); Lago dos Tigres, Station 8, plankton, 16-X-2004, Nogueira et al. 1030 (UFG29925, permanent slide); Lago dos Tigres, Station 7, plankton, 13-VII-2004, Nogueira et al. 903 (UFG29886, permanent slide); Rio Água Limpa, Station 1, plankton, 14-VII-2004, Nogueira et al. 908 (UFG29891, permanent slide); Lago dos Tigres, Station 9, plankton, 17-X-2004, Nogueira et al. 1031 (UFG29926, permanent slide).

Aulacoseira granulata var. valida (Hustedt) Simonsen, Bacillaria 2: 59. 1979.

Figure 7

Frustules cylindrical, forming straight chains united by short linking spines, with short and long separations. Areola striae roughly parallel to, and slightly oblique, in relation to the pervalvar axis. Height: $13-14 \mu \mathrm{m}$; diameter: $11-12 \mu \mathrm{m}$; 9-10 striae in $10 \mu \mathrm{m}$; 8-9 areolae in $10 \mu \mathrm{m}$.

The specimens analyzed demonstrated grossly areola striae, a characteristic that differed Aulacoseira granulata var. valida from variety granulata. The individuals evaluated were similar to those illustrated by Krammer \& Lange-Bertalot (1997, page 266, plate 18 , figure 4). This species was encountered in both lotic and lentic environments.

Geographical distribution in midwestern Brazil: first record.

Occurrence in the samples: BRAZIL. GoIÁs: Britânia, Rio Vermelho, Station 10, plankton, 14-VII2004, Nogueira et al. 906 (UFG29889, permanent slide); Rio Água Limpa, Station 1, plankton, 14-VII-2004, Nogueira et al. 908 (UFG29891, permanent slide); Lago dos Tigres, Station 9, plankton, 17-X-2004, Nogueira et al. 1031 (UFG29926, permanent slide).

Aulacoseira herzogii (Lemmermann) Simonsen, Bacillaria 2: 59. 1979.

Figures 8, 32

Frustules cylindrical, forming straight chains united by long spines. Striae slender, inconspicuous using a light microscope, areolae circular. Height: 6-15 $\mu \mathrm{m}$; diameter: 5-6 $\mu \mathrm{m}$.

The specimens analyzed were encountered in both lentic and lotic environments.
Geographical distribution in midwestern Brazil: Rio Araguaia (Nabout et al. 2006).

Occurrence in the samples: BRAZIL. GoIÁs: Britânia, Lago dos Tigres, Station 7, plankton, 13-VII2004, Nogueira et al. 903 (UFG29886, permanent slide); Rio Vermelho, Station 10, plankton, 14-VII-2004, Nogueira et al. 906 (UFG29889, permanent slide); Rio Água Limpa, Station 1, plankton, 14-VII-2004, Nogueira et al. 908 (UFG29891, permanent slide); Rio Vermelho, Station 11, plankton, 14-VII-2004, Nogueira et al. 907 (UFG29890, permanent slide); Lago dos Tigres, Station 4, plankton, 04-VI-2004, Nogueira et al. 877 (UFG29866, permanent slide); Lago dos Tigres, Station 5, plankton, 04-VI-2004, Nogueira et al. 878 (UFG29867, permanent slide); Rio Vermelho, Station 10, plankton, 05-VI-2004, Nogueira et al. 880 (UFG29869, permanent slide); Rio Vermelho, Station 11, plankton, 05-VI-2004, Nogueira et al. 882 (UFG29871, permanent slide); Lago dos Tigres, Station 6, plankton, 16-X-2004, Nogueira et al. 1028 (UFG29923, permanent slide); Lago dos Tigres, Station 3, plankton, 16-X-2004, Nogueira et al. 1025 (UFG29920, permanent slide); Lago dos Tigres, Station 7, plankton, 16-X-2004, Nogueira et al. 1029 (UFG29924, permanent slide); Lago dos Tigres, Station 8, plankton, 16-X-2004, Nogueira et al. 1030 (UFG29925, permanent slide); Lago dos Tigres, Station 6, plankton, 09-IX-2004, Nogueira et al. 989 (UFG29911, permanent slide).

Aulacoseira muzzanensis (Meister) Krammer, Nova Hedwigia 52: 98. 1991.

Figure 9

Frustules cylindrical, forming straight chains. Areola striae parallel. Valves disconnected, terminating in short spines. Height: 7-8 $\mu \mathrm{m}$; diameter: 11-12 $\mu \mathrm{m}$; 11-14 striae in $10 \mu \mathrm{m} ; 13-16$ areolae in $10 \mu \mathrm{m}$.

Aulacoseira muzzanensis measures $8-25 \mu \mathrm{m}$ in diameter, mantle 4-8 $\mu \mathrm{m}$ tall, striae parallel in almost all valves (Krammer \& Lange-Bertalot 1991). This species demonstrated morphological and morphometric characteristics similar to $A$. distans and A. gessneri. Aulacoseira distans, generally has smaller valves (approximately 8-10 $\mu \mathrm{m}$ in diameter) and numerous inconspicuous rimoportulaes visible under a light microscope; A. gessneri have small linking spines and long spines on the last cells of the chain (Tremarin et al. 2011). As no specimens were observed with the long spines characteristic of $A$. gessneri or the rimoportulaes characteristic of $A$. distans, and the morphometric characteristics of the specimens were close to those described for A. muzzanensis, we chose to identify 
the specimens studied as A. muzzanensis. The samples showed similarities to those illustrated for $A$. muzzanensis by Metzeltin et al. (2005, page 245, plate 244, figure 10) and by Krammer \& Lange-Bertalot (1991, page 271, plate 20, figure 7) and they were encountered in both lentic and lotic environments.

Geographical distribution in midwestern Brazil: first record.

Occurrence in the samples: BRAZIL. GoIÁs: Britânia, Lago dos Tigres, Station 7, plankton, 13-VII2004, Nogueira et al. 903 (UFG29886, permanent slide); Lago dos Tigres, Station 8, plankton, 13-VII2004, Nogueira et al. 904 (UFG29887, permanent slide); Rio Vermelho, Station 11, plankton, 14-VII-2004, Nogueira et al. 907 (UFG29890, permanent slide); Lago dos Tigres, Station 6, plankton, 16-X-2004, Nogueira et al. 1028 (UFG29923, permanent slide); Rio Água Limpa, Station 1, plankton, 16-X-2004, Nogueira et al. 1023 (UFG29918, permanent slide); Lago dos Tigres, Station 3, plankton, 19-VIII-2004, Nogueira et al. 945 (UFG29895, permanent slide); Lago dos Tigres, Station 8, plankton, 19-VIII-2004, Nogueira et al. 950 (UFG29900, permanent slide); Rio Vermelho, Station 11, plankton, 05-VI-2004, Nogueira et al. 881 (UFG29870, permanent slide).

Order Thalassiosirales

Family Stephanodiscaceae

Cyclotella (Kützing) Brébisson

Cyclotella meneghiniana Kutzing, Bacillarien oder Diatomeen: 50. pl. 30. fig. 68. 1844.

Figure 10

Valves circular, central area hyaline, marginal region with radial alveolar striae. Diameter: 8 -19 $\mu \mathrm{m}$; 6-7 striae in $10 \mu \mathrm{m}$.

The population analyzed was distributed throughout the study area and was found in both lotic and lentic environments.

Geographical distribution in midwestern Brazil: Goiânia (Macedo-Saidah et al. 1987, Nogueira \& Leandro-Rodrigues 1999), Planaltina (Souza \& Moreira Filho 1999).

Occurrence in the samples: BRAZIL. GoIÁs: Britânia, Rio Vermelho, Station 10, plankton, 14-VII2004, Nogueira et al. 906 (UFG29889, permanent slide); Lago dos Tigres, Station 4, plankton, 04-VI-2004, Nogueira et al. 877 (UFG29866, permanent slide); Lago dos Tigres, Station 5, plankton, 04-VI-2004, Nogueira et al. 878 (UFG29867, permanent slide); Rio Vermelho, Station 11, plankton, 14-VII-2004, Nogueira et al.
907 (UFG29890, permanent slide); Rio Água Limpa, Station 1, plankton, 14-VII-2004, Nogueira et al. 908 (UFG29891, permanent slide); Rio Vermelho, Station 11, plankton, 05-VI-2004, Nogueira et al. 881 (UFG29870, permanent slide); Lago dos Tigres, Station 2, plankton, 16-X-2004, Nogueira et al. 1024 (UFG29919, permanent slide); Lago dos Tigres, Station 7, plankton, 16-X-2004, Nogueira et al. 1029 (UFG29924, permanent slide); Lago dos Tigres, Station 8, plankton, 16-X-2004, Nogueira et al. 1030 (UFG29925, permanent slide); Rio Vermelho, Station 10, plankton, 17-X-2004, Nogueira et al. 1032 (UFG29927, permanent slide).

\section{Discostella Houk \& Klee}

Discostella stelligera var. stelligera (Cleve \& Grunow) Houk \& Klee, Diatom Research 19 (2): 208. 2004.

Figures 11-12

Valves circular, central area with isolated alveolar striations surrounded by radial alveolar striae of various sizes, forming a rosette, marginal region with simply radiating alveolar striae. Diameter: 7-8 $\mu \mathrm{m} ; 9-14$ striae in $10 \mu \mathrm{m}$.

The specimens analyzed had morphological characteristics similar to those described for $D$. stelligera by Houk \& Klee (2004). The specimens of $D$. stelligera and D. pseudostelligera (Hustedt) Houk \& Klee had marginal processes (fultoportula) in the region of the mantle, although they were different from those of D. pseudostelligera in being extended externally in relatively long tubes that were sometimes bifurcated (which facilitated observing these structures under an optical microscope) (Ferrari \& Ludwig 2007). Discostella woltereckii (Hustedt) Houk \& Klee also had star-shaped alveolus in the central area as did D. stelligera and D. pseudostelligera, although it differed from $D$. stelligera by having a flat central area, while $D$. stelligera is convex, and by having ramified alveolar marginal striae; it likewise differed from $D$. pseudostelligera by having an indistinct central area, while that of $D$. pseudostelligera is flat and very evident (Houk \& Klee 2004).

The numbers of striae in the specimens studied differed from those described for the Brazilian specimens by Fontana \& Bicudo (2008) for the samples (14-17 striae) encountered in superficial sentiments in reservoirs of the Paranapanema River (SP/PR), and by Ferrari \& Ludwig (2007) for specimens (16-18 striae) from the Ivaí River (SP). Discostella stelligera was found in both lentic and lotic environments in the present study.

Geographical distribution in midwestern Brazil: first record. 
Occurrence in the samples: BRAZIL. GoIÁs: Britânia, Rio Vermelho, Station 10, plankton, 14-VII2004, Nogueira et al. 906 (UFG29889, permanent slide); Britânia, Rio Vermelho, Station 11, plankton, 14-VII2004, Nogueira et al. 907 (UFG29890, permanent slide); Lago dos Tigres, Station 5, plankton, 09-IX-2004, Nogueira et al. 989 (UFG29911, permanent slide); Lago dos Tigres, Station 6, plankton, 19-VIII-2004, Nogueira et al. 948 (UFG29898, permanent slide).

Order Triceratiales

Family Triceratiaceae

Pleurosira (Meneghini) Trevisan

Pleurosira laevis (Ehrenberg) Compère, Bacillaria, v. 5, p. 117-178, fig. 1-17, 20, 39. 1982.

Figure 13

Valves circular to sub-circular, valve surface flat with two marginal and opposite ocelli. Areolae in radial rows irregularly distributed from the margin to the center of the valve. Larger diameter: $57-65 \mu \mathrm{m}$; smaller diameter: $50-51 \mu \mathrm{m}$; $15-16$ striae in $10 \mu \mathrm{m}$; 16-17 areolae in $10 \mu \mathrm{m}$.

Pleurosira laevis was encountered only in lotic environments.

Geographical distribution in midwestern Brazil: first record.

Occurrence in the samples: BRAZIL. GoIÁs: Britânia, Rio Água Limpa, Station 1, plankton, 14-VII2004, Nogueira et al. 908 (UFG29891, permanent slide); Rio Vermelho, Station 11, plankton, 14-VII-2004, Nogueira et al. 907 (UFG29890, permanent slide); Rio Vermelho, Station 11, plankton, 05-VI-2004, Nogueira et al. 881 (UFG29870, permanent slide); Rio Vermelho, Station 11, plankton, 05-VI-2004, Nogueira et al. 882 (UFG29871, permanent slide).

Class Fragilariophyceae Round

Order Fragilariales Silva

Family Fragilariaceae Greville

Fragilaria Lyngbye

Fragilaria capucina var. fragilarioides (Grun.) Ludwig \& Flores, Hoehnea 24: 55-65. 1997.

Figures 14-15, 30-31

Valves linear to linear-lanceolate, extremities cuneate subcapitate. Axial area linear, central area circular to rectangular with intumescence of the median region. Parallel striae intercalated at the edges of the valves. Apical axis: $32-40 \mu \mathrm{m}$; transapical axis: $3 \mu \mathrm{m}$; 12-14 striae in $10 \mu \mathrm{m}$.
The specimens from the population analyzed had bilateral intumescence and a rough pattern of striations, characteristics that differentiated F. capucina var. fragilarioides from $F$. capucina var. capucina Desmazières (Ludwig \& Flôres 1997, Brassac \& Ludwig 2003). These taxa were found in lentic as well as lotic environments.

Geographical distribution in midwestern Brazil: first record.

Occurrence in the samples: BRAZIL. GoIÁs: Britânia, Lago dos Tigres, Station 4, plankton, 04-VI2004, Nogueira et al. 877 (UFG29866, permanent slide); Lago dos Tigres, Station 5, plankton, 04-VI-2004, Nogueira et al. 878 (UFG29867, permanent slide); Rio Água Limpa, Station 1, plankton, 14-VII-2004, Nogueira et al. 908 (UFG29891, permanent slide); Lago dos Tigres, Station 5, plankton, 09-IX-2004, Nogueira et al. 989 (UFG29911, permanent slide).

Fragilaria delicatissima (W.Smith) Lange-Bertalot var. delicatissima, Nova Hedwigia 33 (3-4): 723. 1980.

Figure 16

Valves linear to lanceolate, extremities subcapitate, cuneate to acuminate. Axial area linear, central area circular to rectangular, with slight timidity in the median region. Thin parallel striae that intercalate with the margins of the valve. Apical axis: $57-120 \mu \mathrm{m}$; transapical axis: $2-3 \mu \mathrm{m} ; 15-18$ striae in $10 \mu \mathrm{m}$.

The determination of the specimens as F. delicatissima was based on their similarity to the morphological and morphometric characteristics presented by Krammer \& Lange-Bertalot (1997). This taxon was also recorded in Brazil in various rivers in Paraná State by Brassac \& Ludwig (2003). The specimens of $F$. delicatissima var. delicatissima were encountered in both lentic and lotic environments in the present study.

Geographical distribution in midwestern Brazil: first record.

Occurrence in the samples: BRAZIL. GoIÁs: Britânia, Lago dos Tigres, Station 5, plankton, 04-VI2004, Nogueira et al. 878 (UFG29867, permanent slide); Lago dos Tigres, Station 8, plankton, 05-VI2004, Nogueira et al. 879 (UFG29868, permanent slide); Lago dos Tigres, Station 5, plankton, 09-IX-2004, Nogueira et al. 989 (UFG29911, permanent slide); Lago dos Tigres, Station 6, plankton, 16-X-2004, Nogueira et al. 1028 (UFG29923, permanent slide); Lago dos Tigres, Station 7, plankton, 16-X-2004, Nogueira et al. 1029 (UFG29924, permanent slide); Rio Vermelho, Station 11, plankton, 05-VI-2004, Nogueira et al. 
881 (UFG29870, permanent slide); Rio Vermelho, Station 11, plankton, 14-VII-2004, Nogueira et al. 906 (UFG29889, permanent slide); Lago dos Tigres, Station 6, plankton, 19-VIII-2004, Nogueira et al. 948 (UFG29898, permanent slide).

Fragilaria javanica Hustedt, Archiv für Hydrobiologie 6(1): 154, pl.10, figs. 59-60. 1938.

Figures 17-18

Valves linear, extremities subcapidate to capitate. Axial and central areas absent; parallel striae along all of the valvar surface. Apical axis: 45-120 $\mu \mathrm{m}$, transapical axis: 5-6 $\mu \mathrm{m}, 20$ striae in $10 \mu \mathrm{m}$.

Fragilaria javanica is distinguishable from Fragilariforma virescens (Ralfs) Williams \& Round by not showing a narrow axial area and extremities rostrate (Brassac \& Ludwig 2003); their valvar shapes are very similar, however. This species was present in both lentic and lotic environments.

Geographical distribution in midwestern Brazil: first record.

Occurrence in the samples: BRAZIL. GoIÁs: Britânia, Lago dos Tigres, Station 4, plankton, 04-VI2004, Nogueira et al. 877 (UFG29866, permanent slide); Lago dos Tigres, Station 5, plankton, 04-VI-2004, Nogueira et al. 878 (UFG29867, permanent slide); Lago dos Tigres, Station 6, plankton, 16-X-2004, Nogueira et al. 1028 (UFG29923, permanent slide); Rio Vermelho, Station 11, plankton, 06-XI-2004, Nogueira et al. 1025 (UFG29939, permanent slide); Lago dos Tigres, Station 5, plankton, 09-IX-2004, Nogueira et al. 989 (UFG29911, permanent slide).

\section{Staurosira Ehrenberg}

Staurosira acutirostrata (Metzeltin \& Lange-Bertalot) Metzeltin \& Lange-Bertalot, In Metzeltin et al. Iconographia Diatomologica 15: pl. 13, fig. 21, 2005. Figures 19-20

Valves lanceolate, projected valvar extremities rounded to cuneate. Axial area lanceolate. Striae rough, arranged alternately. Apical axis: 18-25 $\mu \mathrm{m}$; transapical axis: $5-7 \mu \mathrm{m}$; 8 striae in $10 \mu \mathrm{m}$.

The specimens analyzed had less tapered extremities, although they were morphologically similar to those described for Staurosira acutirostrata (apical axis (14)22$36 \mu \mathrm{m}$, transapical axis 4-6 $\mu \mathrm{m}$, and 7-9 striae in $10 \mu \mathrm{m})$ and illustrated by Metzeltin \& Lange-Bertalot (1998, page 236, plate 1, figures 18-19). The specimens differed from Staurosirella crassa (D.Metzeltin \& H.LangeBertalot) Ribeiro \& Torgan by having more elongated apices, smaller valvar proportions, and greater numbers of striae in $10 \mu \mathrm{m}$; differing from Staurosira pinnata Ehrenberg by having larger apical axes. Staurosira acutirostrata was encountered in the present study in both lentic and lotic environments.

Geographical distribution in midwestern Brazil: first record.

Occurrence in the samples: BRAZIL. GoIÁs: Britânia, Rio Água Limpa, Station 1, plankton, 14-VII2004, Nogueira et al. 908 (UFG29891, permanent slide); Lago dos Tigres, Station 4, plankton, 04-VI-2004, Nogueira et al. 877 (UFG29866, permanent slide); Rio Água Limpa, Station 1, plankton, 09-IX-2004, Nogueira et al. 985 (UFG29907, permanent slide).

Staurosirella Williams \& Round

Staurosirella crassa (D.Metzeltin \& H.Lange-Bertalot) Ribeiro \& Torgan, Revista Brasileira de Paleontologia 13(1): 21-32. 2010.

Figures 21-22

Valves lanceolate, valvar extremities projected, subcapitate, rounded. Axial area linear. Striae arranged alternately. Apical axis: 29-51 $\mu \mathrm{m}$; transapical axis: 11-12 $\mu \mathrm{m}$; 4-7 striae in $10 \mu \mathrm{m}$.

The morphological characteristics of the apical axis and the valvar outline were similar to those described by Metzeltin \& Lange-Bertalot (1998, page 236, plate 1, figures 20-23) for Fragilaria crassa Metzeltin \& Lange-Bertalot. These taxa have valvar shapes similar to Staurosira acutirostrata, although they differ in having greater valvar sizes and larger numbers of striae. This species was encountered in both lentic and lotic environments.

Geographical distribution in midwestern Brazil: first record.

Occurrence in the samples: BRAZIL. GoIÁs: Britânia, Lago dos Tigres, Station 4, plankton, 04-VI2004, Nogueira et al. 877 (UFG29866, permanent slide); Lago dos Tigres, Station 5, plankton, 04-VI2004, Nogueira et al. 878 (UFG29867, permanent slide); Rio Vermelho, Station 11, plankton, 14-VII2004, Nogueira et al. 907 (UFG29890, permanent slide); Rio Água Limpa, Station 1, plankton, 09-IX-2004, Nogueira et al. 985 (UFG29907, permanent slide); Lago dos Tigres, Station 6, plankton, 16-X-2004, Nogueira et al. 1028 (UFG29923, permanent slide); Lago dos Tigres, Station 7, plankton, 13-VII-2004, Nogueira et al. 903 (UFG29886, permanent slide); Rio Água Limpa, Station 1, plankton, 20-VIII-2004, Nogueira et al. 954 (UFG29904, permanent slide). 

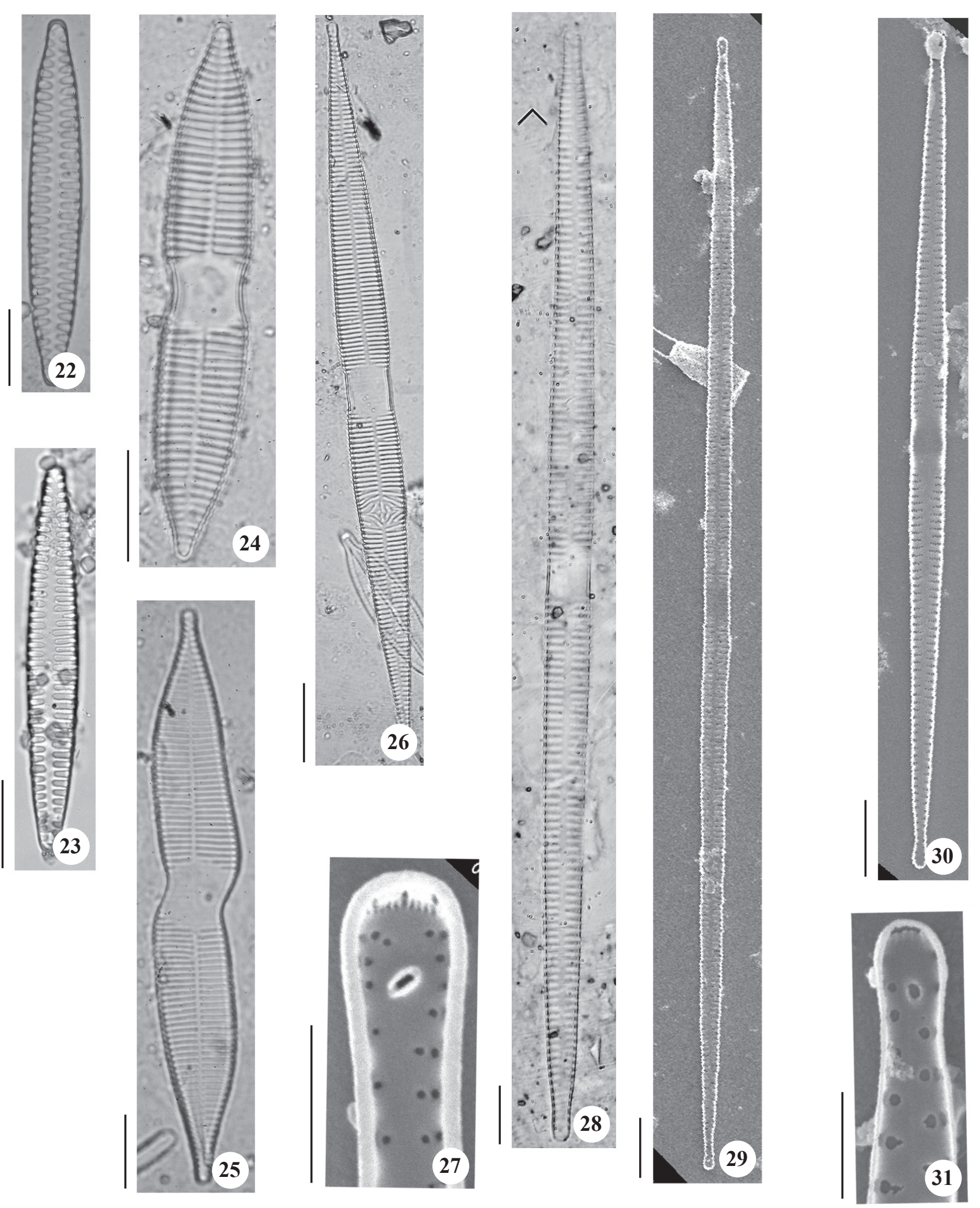

Figures 22-31. Planktonic Coscinodiscophyceae and Fragilariophyceae in the Lago dos Tigres hydrologic system, Goiás, Brazil. 22-26 and 28. Valvar view using an optical microscope. 27 and 29-31. Valvar view using a scanning electron microscope. 22. Staurosirella crassa. 23. Staurosirella sp.1. 24-26. Synedra goulardii var. goulardii. 27-29. Ulnaria ulna. 30-31. Fragilaria capucina var. fragilarioides. Bar $=10 \mu \mathrm{m}(22-26,28-30) ; 1 \mu \mathrm{m}(27,31)$. 


\section{Staurosirella $\mathrm{sp} .1$}

Figure 23

Valves lanceolate, valvar extremities rounded, rostrate to cuneate. Axial area linear. Striae alternately arranged. Apical axis: $87.5-90 \mu \mathrm{m}$; transapical axis: 13-14 $\mu \mathrm{m}$; 4 striae in $10 \mu \mathrm{m}$.

The valves of Staurosirella sp.1 were similar to those of Staurosirella crassa (D. Metzeltin \& H. LangeBertalot) Ribeiro \& Torgan, although they were strongly lanceolate with extremities rostrate; the valvar axis greater than $50 \mu \mathrm{m}$ distinguished the former species. The genus Staurosirella differs from Fragilaria by having atriae composed of linear areolae, and rimoporulae were not observed. The thick ribs separating the areolae give genus Staurosirella a rougher valvar striation pattern than seen in the genus Staurosira (Williams \& Round 1987, Round 1991), and the wider inter-striae than striae areas also distinguish the former from the latter genus. This taxon was only encountered in lentic environments.

Occurrence in the samples: BRAZIL. GoIÁs: Britânia, Lago dos Tigres, Station 8, plankton, 16-X-2004, Nogueira et al. 1030 (UFG29925, permanent slide).

\section{Synedra Ehrenberg}

Synedra goulardii Brébisson var. goulardii, In Cleve \& Grunow, Kongliga Svenska Vetenskaps-Akademiens Handlingar 17(2): 107, pl. 6, fig. 119. 1880.

Figures 24-26

Valves linear-lanceolate to lanceolate, constricted in the median region; extremities subcapitate, rounded to cuneate. Axial area linear, central area rectangular to circular. Striae parallel and approximately aligned with those on the opposite margin. Apical axis: 79-120 $\mu \mathrm{m}$; transapical axis: 7-8 $\mu \mathrm{m}$; 9-16 striae in $10 \mu \mathrm{m}$.

The population studied appeared to be polymorphic, with individuals with subtle to strong valvar restrictions in the median region. Some specimens showed malformations. Synedra goulardii was only encountered in lotic environments.

Geographical distribution in midwestern Brazil: Goiânia (Macedo-Saidah et al. 1987, Campos \& MacedoSaidah 1990, Brandão \& Kravchenko 1997).

Occurrence in the samples: BRAZIL. GoIÁs: Britânia, Rio Vermelho, Station 11, plankton, 05-VI-2004, Nogueira et al. 881 (UFG29870, permanent slide); Rio Vermelho, Station 11, plankton, 17-X-2004, Nogueira et al. 1033 (UFG29928, permanent slide); Rio Vermelho, Station 11, plankton, 14-VII-2004, Nogueira et al. 907 (UFG29890, permanent slide); Rio Água Limpa, Station 1, plankton, 14-VII-2004, Nogueira et al. 908 (UFG29891, permanent slide).

\section{Ulnaria (Kützing) Compère}

Ulnaria ulna (Nitzsch) Compère, Jahn et al. Studies on Diatoms, p. 100. 2001.

Figures 27-29

Valves linear to linear-lanceolate, without restriction in the median region. Extremities rounded to rostrate subcapitate. Axial area linear, central area circular to rectangular. Striae parallel. Apical axis: 100-200 $\mu \mathrm{m}$; transapical axis: $5-8 \mu \mathrm{m}$; 8-9 striae in $10 \mu \mathrm{m}$.

Ulnaria ulna was encountered in lentic and lotic environments.

Geographical distribution in midwestern Brazil: first record.
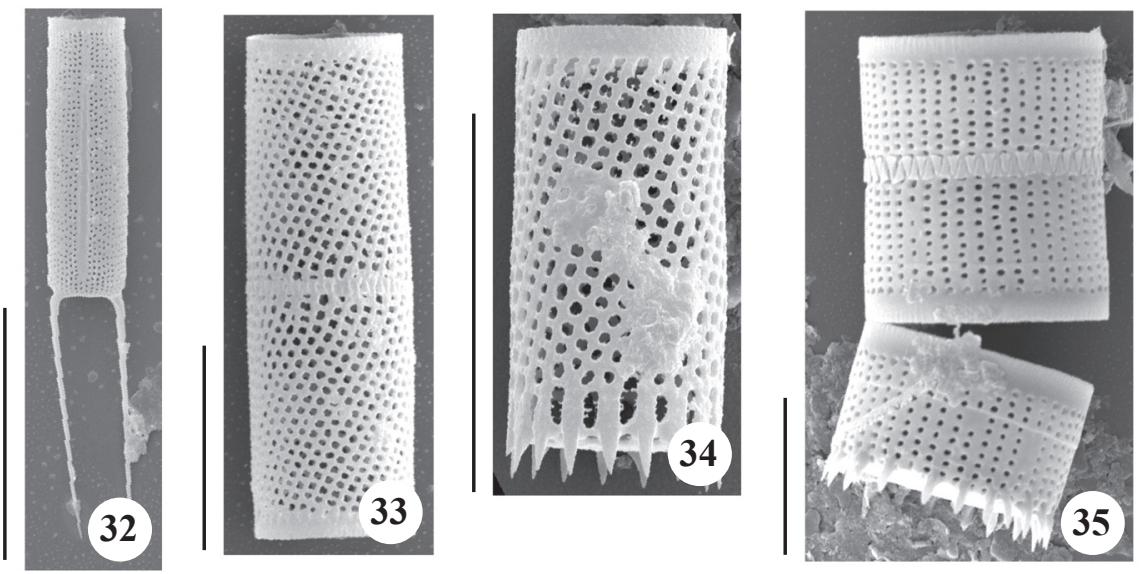

Figures 32-35. Planktonic Coscinodiscophyceae and Fragilariophyceae in the Lago dos Tigres hydrologic system, Goiás, Brazil. Valvar view using a scanning electron microscope. 32. Aulacoseira herzogii. 33-34. Aulacoseira ambigua. 35. Aulacoseira distans. Bar $=10 \mu \mathrm{m}$. 
Occurrence in the samples: BRAZIL. GoIÁs: Britânia, Rio Vermelho, Station 10, plankton, 05-VI2004, Nogueira et al. 880 (UFG29869, permanent slide); Rio Vermelho, Station 11, plankton, 05-VI-2004, Nogueira et al. 881 (UFG29870, permanent slide); Lago dos Tigres, Station 4, plankton, 04-VI-2004, Nogueira et al. 877 (UFG29866, permanent slide); Lago dos Tigres, Station 5, plankton, 04-VI-2004, Nogueira et al. 878 (UFG29867, permanent slide); Rio Água Limpa, Station 1, plankton, 20-VIII-2004, Nogueira et al. 954 (UFG29904, permanent slide); Lago dos Tigres, Station 5, plankton, 09-IX-2004, Nogueira et al. 989 (UFG29911, permanent slide); Rio Água Limpa, Station 1, plankton, 09-IX-2004, Nogueira et al. 985 (UFG29907, permanent slide); Lago dos Tigres, Station 3, plankton, 16-X-2004, Nogueira et al. 1025 (UFG29920, permanent slide); Lago dos Tigres, Station 4, plankton, 13-VII-2004, Nogueira et al. 900 (UFG29882, permanent slide); Lago dos Tigres, Station 6, plankton, 13-VII-2004, Nogueira et al. 902 (UFG29884, permanent slide); Lago dos Tigres, Station 2, plankton, 13-VII-2004, Nogueira et al. 898 (UFG29880, permanent slide); Lago dos Tigres, Station 2, plankton, 05-XI-2004, Nogueira et al. 1116 (UFG29930, permanent slide); Lago dos Tigres, Station 6, plankton, 19-VIII-2004, Nogueira et al. 948 (UFG29898, permanent slide).

Acknowledgments - The authors thank the Secretaria de Ciência e Tecnologia do Estado de Goiás (SECTEC/GO); the Laboratório Multiusuário de Microscopia de Alta Resolução (Lammar) of the Instituto de Física / Universdade Federal de Goiás, the CNPq for financing this project (Proc. 23234075); and the Coordenação de Aperfeiçoamento de Pessoal de Nível Superior (Capes) for the Masters grant awarded to the first author.

\section{REFERENCES}

Bicudo CEM, Menezes M. 2006. Gêneros de algas de águas continentais do Brasil: chave de identificação e descrições. RiMa, São Carlos.

Brandão D, Kravchenko A. 1997. A biota do Campus Samambaia: história, situação e perspectivas. SEGRAFUFG, Goiânia.

Brassac NM, Ludwig TAV. 2003. Fragilariaceae (Bacillariophyceae) de rios da bacia do Iguaçu, Estado do Paraná, Brasil. Revista Brasileira de Botânica 26: 311-318.

Campos IFP, Macedo-Saidah FF. 1990. Flórula da represa da escola de agronomia da Universidade Federal de Goiás, Goiânia, Goiás, Brasil. In Anais do XXXVI Congresso Nacional de Botânica. Ibama/SBB, Brasília, p.839-857.
Ferrari F, Ludwig TAV. 2007. Coscinodiscophyceae, Fragilariophyceae e Bacillariophyceae (Achnanthales) dos rios Ivaí, São João e dos Patos, bacia hidrográfica do rio Ivaí, município de Prudentópolis, PR, Brasil. Acta Botanica Brasilica 21:421-441.

Geogoiás. 2002. O estado ambiental de Goiás. Agência Ambiental de Goiás/Fundação CEBRAC/PNUMA/ SEMARH, Brasília.

Fontana L, Bicudo DC. 2009. Diatomáceas (Bacillariophyceae) de sedimentos superficiais dos reservatórios em cascata do Rio Paranapanema(SP/PR, Brasil): Coscinodiscophyceae e Fragilariophyceae. Hoehnea 36:375-386.

Houk V, Klee R. 2004. The stelligeroid taxa of the genus Cyclotella (Kützing) Brébisson (Bacillariophyceae) and their transfer into the new genus Discostella gen. nov. Diatom Research 19:203-228.

KrammerK,Lange-BertalotH.1991.Bacillariophyceae.3.Teil: Centrales, Fragilariaceae, Eunotiaceae. Süsswasserflora von Mittleleuropa. G Fischer, Stuttgart v.1.

KrammerK,Lange-BertalotH.1997.Bacillariophyceae.3.Teil: Centrales, Fragilariaceae, Eunotiaceae. Süsswasserflora von Mittleleuropa. G Fischer, Stuttgart, v.2.

Landucci M, Ludwig TAV. 2005. Diatomáceas de rios da bacia hidrográfica litorânea, PR, Brasil: Coscinodiscophyceae e Fragilariophyceae. Acta Botanica Brasilica 19:345-357.

Ludwig TAV, Bigunas PTI. 2006. Bacillariophyta. In Gêneros de algas de águas continentais do Brasil: chave de identificação e descrições. (CEM Bicudo, M. Menezes, eds.). RiMa, São Carlos. p.391-439.

Ludwig TAV, Flôres TL, Moreira Filho H, Veiga LAS. 2004. Inventário florístico das diatomáceas (Ochrophyta) de lagoas do Sistema Hidrológico do Taim, Rio Grande do Sul, Brasil: Coscinodiscophyceae. Iheringia 59:97-106.

Ludwig TAV, Flôres T. 1997. Diatomoflórula dos rios da região a ser inundada para a construção da Usina Hidrelétrica de Segredo, Paraná: Fragilariophyceae (Fragilaria e Synedra). Hoehnea 24:55-65.

Macedo-Saidah FEM, Nascimento MRR, Campos IEP. 1987. O plâncton das águas do Rio Meia Ponte, Município de Goiânia, Goiás, Brasil. Nerítica 2:105-117.

Metzeltin D, Lange-Bertalot H. 1998. Tropical diatoms of South America I. Iconographia diatomologica. Koeltz Scientific Books, Königstein, v.5.

Morales EA. 2001. Morphological studies in selected fragilarioid diatoms (Bacillariophyceae) from Connecticut waters (U.S.A). Proceedings of the Academy of Natural Sciences of Philadelphia 151:105-120.

Morales EA. 2005. Observations of the morphology of some known and new fragilarioid diatoms (Bacillariophyceae) from rivers in the USA. Phycological Research 53: 113-133.

Morales EA, Le M. 2005. A new species of the diatom genus Adlafia (Bacillariophyceae) from the United States. Proceedings of the Academy of Natural Sciences of Philadelphia 154:149-154. 
Morales EA, Manyolov KM, Bahls LL. 2010. Three new araphid diatoms (Bacillariophyta) from rivers in North America. Proceedings of the Academy of Natural Sciences of Philadelphia 160:29-46.

Morandi LL, Ritter LMO, Moro RS, Bicudo CEM. 2006. Criptógamos do Parque Estadual das Fontes do Ipiranga, São Paulo, SP. Algas, 20: Coscinodiscophyceae. Hoehnea 33:115-122.

Moreira Filho H, Valente-Moreira IM. 1981. Avaliação taxonômica e ecológica das diatomáceas (Bacillariophyceae) epífitas em algas pluricelulares obtidas nos litorais dos estados do Paraná, Santa Catarina e São Paulo. Boletim do Museu Botânico Municipal 47:1-17.

Nabout JC, Nogueira IS, Oliveira LG. 2006. Phytoplankton community of flooplain lakes of the Araguaia River, Brazil, in the rainy and dry seasons. Journal of Plankton Research 28:181-193.

Nabout JC, Nogueira IS. 2007. Spatial and temporal dynamics of phytoplankton functional group in a blocked valley (Brazil). Acta Limnologica Brasiliensia 19:305-314.

Nogueira IS, Leandro-Rodrigues NC. 1999. Algas planctônicas do Lago do Jardim Botânico Chico Mendes, GoiâniaGO: considerações taxonômicas e ecológicas. Revista Brasileira de Biologia 59:377-395.
Potapova MG, Charles DF. 2003. Distribution of benthic diatoms in U.S. rivers in relation to conductivity and ionic composition. Freshwater Biology 48:1311-1328.

Round FE. 1991. Use of diatoms for monitoring rivers. In Use of algae for monitoring rivers (BA Whitton, E Rott, G Friedrich, eds.). Institut für Botanik/Universität Innsbruck, Innsbruck, p.25-32.

Round FE, Crawford RM, Mann DG. 1990. The diatoms: biology and morphology of the genera. Cambridge University Press, Cambridge.

Simonsen, R. 1974. The diatom plankton of the Indian Ocean expedition of R/V "Meteor". Meteor-forscheingsergeb Reihe D, Biologie 19:1-66.

Souza MGM, Moreira Filho H. 1999. Diatoms (Bacillariophyceae) of two aquatic macrophyte banks from Lagoa Bonita, Distrito Federal, Brazil, I: Thalassiosiraceae and Eunotiaceae. Bulletin du Jardin Botanique National de Belgique 67: 259-278.

Tremarin P, Loverde-Oliveira S, Ludwig TV, Torgan LC. 2011. Ultrastructure and distribution of Aulacoseira gessneri. Diatom Research 26:189-197.

Wetzel RG, Likens GE. 1991. Limnological analysis. 2nd ed., Springer-Verlag, New York.

Williams DM, Round FE. 1987. Revision of the genus Fragilaria. Diatom Research 2:267-288. 